\title{
Mobilferrin Is an Intermediate in Iron Transport between Transferrin and Hemoglobin in K562 Cells
}

\author{
Marcel E. Conrad, Jay N. Umbreit, Elizabeth G. Moore, and David Heiman \\ University of South Alabama, Mobile, Alabama 36688
}

\begin{abstract}
Iron is bound to transferrin in the plasma. A specific receptor on the cell surface binds transferrin and internalizes transferrin and the iron in clathrin-coated pits. These invaginate to form vesicles which release iron to the cytoplasm. Inorganic iron can be transported by an alternative pathway from iron citrate, utilizing a cell surface integrin and a cytoplasmic protein mobilferrin. This article shows that the two pathways donate iron to mobilferrin which acts as an intermediate between the iron bound to transferrin and the incorporation of iron into hemoglobin. Mobilferrin is found associated with the transferrin containing vesicles, and becomes labeled with iron released from transferrin in the vesicles. Mobilferrin is also found in the cytoplasm where pulse-chase experiments show that it, in turn, releases iron to be used for the synthesis of hemoglobin. (J. Clin. Invest. 1996. 98:1449-1454.) Key words: calreticulin • paraferritin • clathrin $\bullet$ pulse-chase $\bullet$ endosome
\end{abstract}

\section{Introduction}

Iron is transported into mammalian cells via two described pathways. Most plasma iron is transported to cells in the body bound to transferrin. Cell membranes possess a transferrin receptor that binds the transferrin and invaginates into the cell to form a vesicle $(1,2)$. Acidification of the contents of the vesicle releases iron from transferrin so that it is delivered to the cytoplasm. The vesicle then returns to the surface of the cell to deliver apotransferrin to the plasma $(3,4)$.

A second pathway was sought in small intestinal absorptive cells because there are no transferrin receptors in microvilli (5). This pathway absorbs soluble iron chelates, such as ferric citrate. The iron associates with a cell surface integrin $(6,7)$ and subsequently is transferred to a cytosolic protein named mobilferrin (8).

Many investigators have reported that there is an alternative pathway for iron uptake in nonintestinal cells (9-20). Recently, it was shown in K562 and Raji cells that this pathway utilizes integrin and mobilferrin similar to intestinal cells (21). Further,

Address correspondence to Marcel E. Conrad M.D., USA Cancer Center, University of South Alabama, Mobile, AL 36688. Phone: 334460-7194; FAX: 334-460-7637.

Received for publication 29 April 1996 and accepted in revised form 12 July 1996.

J. Clin. Invest.

(C) The American Society for Clinical Investigation, Inc.

0021-9738/96/09/1449/06 \$2.00

Volume 98, Number 6, September 1996, 1449-1454 chase experiments in K562 erythroleukemia cells suggested there might be an interrelationship between the two pathways because some radioiron presented to the cells bound to transferrin was isolated from the cytosol complexed to mobilferrin (21).

This study explores the postulated interrelationship between the transferrin-transferrin receptor pathway and the mobilferrin-integrin pathway (MIP) ${ }^{1}$ Utilizing pulse chase experiments and Western blot analysis of membrane fractions isolated from K562 cells, sequential labeling of mobilferrin and hemoglobin was demonstrated in cells in which iron was transported to the cytosol bound to transferrin. Further, a close association of mobilferrin and transferrin containing vesicles was demonstrated in the cytosol of K562 cells. These findings suggested that mobilferrin is a component of both pathways.

\section{Methods}

Separation of membrane components. K562 cells were selected for these studies because they possess both the transferrin-transferrin receptor and the MIP pathway to transport iron into the cell (21). Further, they synthesize hemoglobin at a slow rate which permitted identification of intermediate carriers of iron in pulse chase experiments when radioiron with a high specific activity $(20 \mathrm{mCi} / \mathrm{mg} \mathrm{Fe})$ was utilized. Human apotransferrin (Sigma Chemical Co., St. Louis, MO) was labeled with $2 \mathrm{~mol}$ of ${ }^{59} \mathrm{Fe}-\mathrm{NTA}$ (Dupont, Irving, TX) per mole of transferrin. Excess radioiron was removed by filtration (Centricon10, Amicon, Danver, MA). K562 cells were grown in RPMI 1640 (GIBCO, Gaithersburg, MD) with 10\% FCS in spinner flasks and collected by centrifugation. The cells were washed with $10 \mathrm{mM}$ Hepes, $0.15 \mathrm{M} \mathrm{NaC} 1,0.1 \%$ glucose (HSG). The cell number was determined with a hemocytometer and viability was determined by Trypan blue exclusion. An aliquot of cells $\left(12.5 \times 10^{7}\right.$ cells $)$ was incubated with $0.25 \mathrm{mg}$ of transferrin in $25 \mathrm{ml}$ of $\mathrm{HSG}$ at $4^{\circ} \mathrm{C}$ for $30 \mathrm{~min}$. This allowed the transferrin to saturate the receptor (22-24). The cells were rapidly warmed to $37^{\circ} \mathrm{C}$ for the indicated time. Then the cells were chilled, collected by centrifugation, washed with HSG at $4^{\circ} \mathrm{C}$ and resuspended in $2 \mathrm{ml}$ of $10 \mathrm{mM}$ Hepes pH 7.5 with $10 \mathrm{mM}$ $\mathrm{NaC} 1(\mathrm{HNa})$ and sonicated with $25 \mathrm{~W}$ at $4^{\circ} \mathrm{C}$ for $30 \mathrm{~s}$. The solution was examined microscopically to insure complete cellular rupture had occurred and the suspension was applied to a $60-20 \%$ (W/W) linear sucrose gradient (24) in $36 \mathrm{ml}$ polyallomer tubes. Centrifugation was performed in a Sorvall S28 centrifuge with a SS28/36 swingingbucket rotor at 19,000 rpm for $18 \mathrm{~h}$. The tubes were punctured at the base and 1-ml fractions were obtained.

\section{Western blots}

Fractions from the sucrose gradient were subjected to SDS-PAGE by the Laemmli method and transferred to Hybond nitrocellulose (Amersham, Arlington Heights, IL) utilizing a BioRad transblot apparatus (25). The blots were probed with antibody to mobilferrin and developed by an enhanced chemiluminescence method (Amersham).

1. Abbreviations used in this paper: $\mathrm{Hgb}$, hemoglobin; $\mathrm{HNa}, \mathrm{Hepes} \mathrm{sa}-$ line; HSG, Hepes-saline-glucose; Mb, mobilferrin; MIP, mobilferrinintegrin pathway; Tf, transferrin. 


\section{Gel filtration}

An Ultrogel AcA44 column (IBF Biotechnics, Columbia, MD) column $(1.5 \times 50 \mathrm{~cm})$ was equilibrated with $\mathrm{HNa}$. Selected samples from the sucrose gradient were combined with an equal volume of $2 \mathrm{M}$ $\mathrm{NaC} 1$ and incubated at $37^{\circ} \mathrm{C}$ for $10 \mathrm{~min}$. The sample was applied to the column and fractions of $1 \mathrm{ml}$ were obtained and the radioactivity determined. The column was calibrated with authentic samples of protein of known molecular mass. The fractions containing radioactivity corresponding to the elution volume of mobilferrin were pooled and mixed with 1:300 dilutions of antibody to mobilferrin and incubated at $4^{\circ} \mathrm{C}$ for $24 \mathrm{~h} .25 \mu \mathrm{l}$ of Sepharose-protein G (Sigma Chemical Co.) was added, and incubated at $4^{\circ} \mathrm{C}$ overnight. It was collected by centrifugation and washed three times with $\mathrm{HNa}$. Radioactivity on the beads was then quantified in a gamma counter.

\section{Pulse chase}

For each determination, $2 \mathrm{ml}$ of K562 cells $\left(1.9 \times 10^{6}\right)$ was incubated with $20 \mu \mathrm{l}$ of ${ }^{59} \mathrm{Fe}$-radiolabeled diferric human transferrin $(1 \mathrm{mg} / \mathrm{ml})$. After a 10-min incubation at $37^{\circ} \mathrm{C}$ unlabeled differic human transferrin $(200 \mu \mathrm{l}$ of $10 \mathrm{mg} / \mathrm{ml})$ was added and the incubation continued for
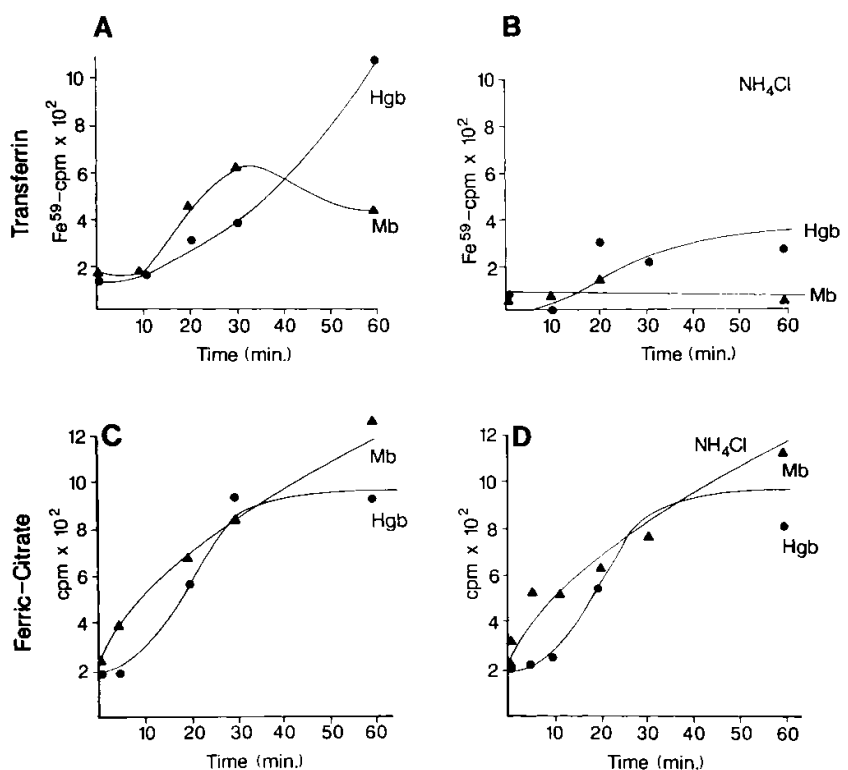

Figure 1. Pulse-chase labeling of mobilferrin. Radiolabeled iron in the form of differic transferrin was incubated with K562 cells for 10 min and then a 25 -fold excess of unlabeled transferrin was added. At the times indicated, the cells were lysed and the cytoplasmic fraction obtained. Immunoprecipitates were found with antibody to human hemoglobin $(\mathrm{Hgb})$ or mobilferrin $(\mathrm{Mb})$. The amount of radiolabeled iron present in the hemoglobin $(\bullet)$ and mobilferrin $(\boldsymbol{\Delta})$ was determined. Lines represent the best fit plot of the temporal measurements. Points are the means of triplicate measurements. $(A)$ The results obtained during incubation in serum free HSG buffer are shown. (B) The addition of $15 \mathrm{mM} \mathrm{NH}_{4} \mathrm{Cl}$, which is known to inhibit the release of transferrin iron from the endosome also remarkably inhibited the labeling of hemoglobin $(\bullet)$ and mobilferrin $(\mathbf{\Lambda})$. (C) Radiolabeled iron was in the form of ferric citrate $\left(10^{-7} \mathrm{M}\right)$ and the chase utilized a 10-fold excess of ferric citrate. Mobilferrin and hemoglobin were assayed as described above. $(D)$ The label was ferric citrate as in $C$, but the incubation was performed with addition of $15 \mathrm{mM} \mathrm{NH}_{4} \mathrm{Cl}$. Hemoglobin $(\bullet)$ and mobilferrin $(\boldsymbol{\Lambda})$ were determined by immunoprecipitation at the indicated times. $\mathrm{NH}_{4} \mathrm{Cl}$ did not affect the transport of iron from ferric citrate; this excludes the possible use of residual transferrin on the surface of cells by ferric citrate to gain entry into the cells.

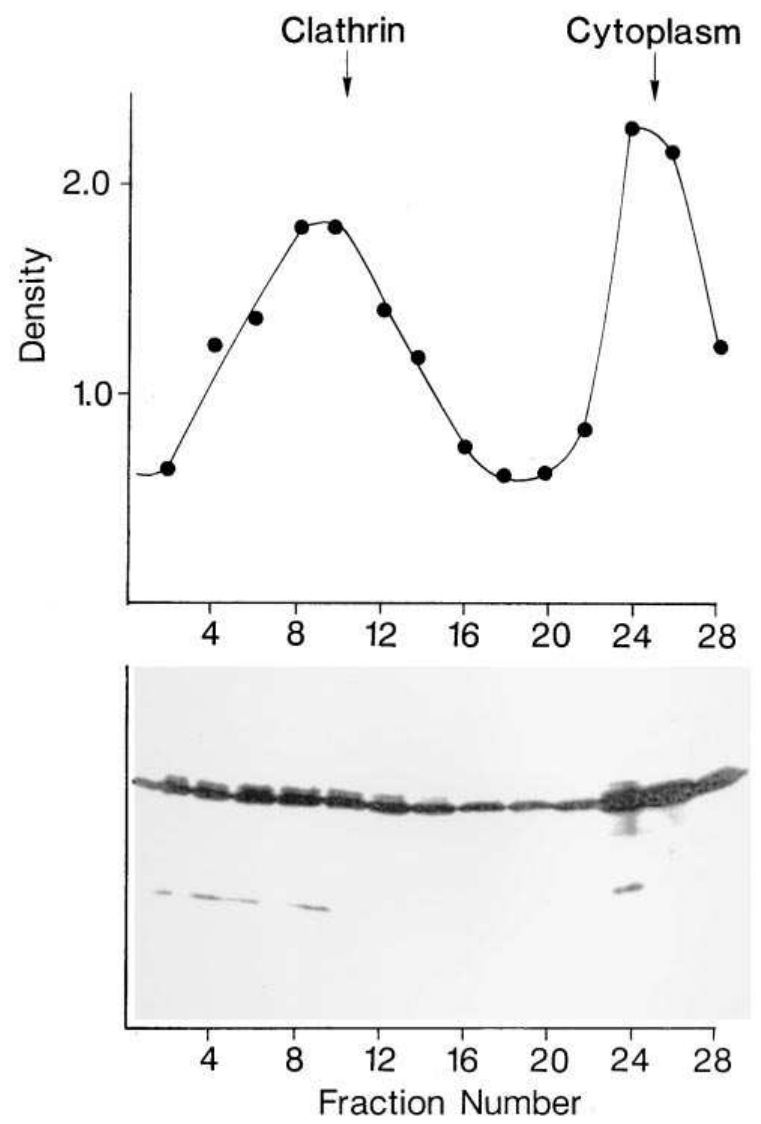

Figure 2. Localization of mobilferrin in the vesicular fraction. K562 cells were lysed and the entire lysate applied to a $60-20 \% \mathrm{~W} / \mathrm{W}$ sucrose gradient, and centrifuged to equilibrium at 19,000 rpm for $18 \mathrm{~h}$. Fractions were then taken and subjected to SDS-PAGE, Western blotted and developed with antibody to mobilferrin. $(A)$ Indicates the densitometry results from the Western blot in the arbitrary units of the immunoreactivity against mobilferrin shown in $B$. The clathrin fraction was localized by the parallel Western blot against clathrin antibody (Sigma, blot, not shown). (B) Western blots of mobilferrin showing its presence in the fractions peaking at tubes 7-12 coincident with the major immunoreactivity of clathrin and in the soluble cytoplasmic fraction at tubes $22-28$. The dense portion of the sucrose gradient is at fraction 1 and the least dense sucrose at fraction 23, with fractions 23-28 representing the portion of cytoplasm not entering the gradient.

various times at $37^{\circ} \mathrm{C}$ in $\mathrm{HSG}$. After incubation the cells were rapidly chilled, collected by centrifugation $(2,000 \mathrm{~g}$ for $10 \mathrm{~min})$, washed twice in HSG, and lysed by freezing and thawing in $1 \mathrm{ml}$ of $\mathrm{HNa}$ buffer followed by sonification ( $25 \mathrm{~W}, 1 \mathrm{~min}$ ). After complete lysis was confirmed by microscopy, the cytosolic fraction was recovered by centrifugation in a microcentrifuge for $15 \mathrm{~min}$. Antibody against either mobilferrin or hemoglobin $(20 \mu \mathrm{l})$ was added to $320 \mu \mathrm{l}$ of the cytosol and incubated at $4^{\circ} \mathrm{C}$ for $4 \mathrm{~h}$. An appropriate secondary antibody (goat anti-rabbit, Sigma Chemical Co.) was added and allowed to form a precipitate overnight. The immunoprecipitate was recovered by centrifugation, washed twice in HSG, and the radioactivity in the precipitate determined for each interval.

Antibody against mobilferrin was obtained as a polyclonal anticalreticulin antibody (Affinity Bioreagants Inc., Golden, CO). Mobilferrin is a homologue of calreticulin and antibodies were shown to be cross-reactive (26). This antibody was chosen because it retains its immunoreactivity after heating at $100^{\circ} \mathrm{C}$ better than our available anti- 

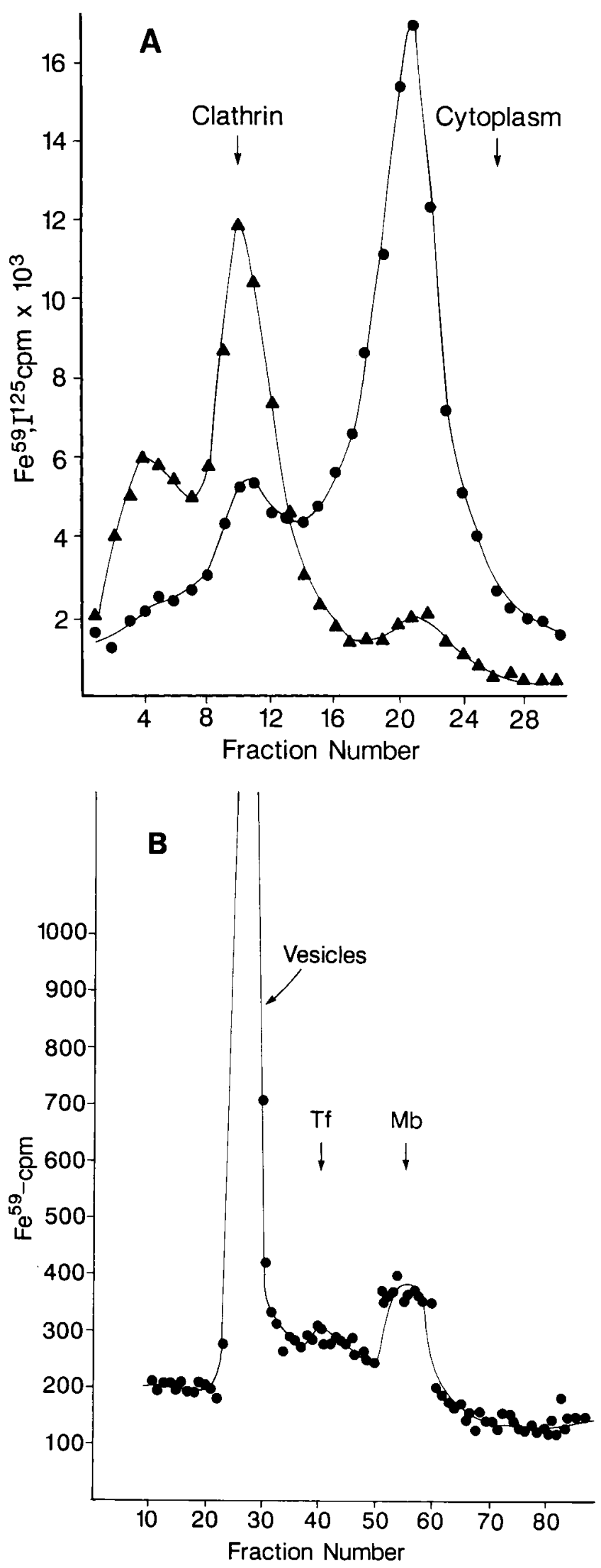

Figure 3. Mobilferrin binds iron released from transferrin $(A) \mathrm{Su}-$ crose gradient of K562 cells incubated with transferrin. The buffers used for the incubation and for the sucrose gradient all contained 15 $\mathrm{mM} \mathrm{NH}_{4} \mathrm{C} 1$. Cells were allowed to bind a mixture of ${ }^{59} \mathrm{Fe}$ diferric mobilferrin antibodies. Antihuman hemoglobin was obtained from Sigma (H4890).

\section{Results}

Transferrin conveys iron to mobilferrin. K562 cells were incubated with ${ }^{59} \mathrm{Fe}$ radiolabeled diferric transferrin for $10 \mathrm{~min}$ at $37^{\circ} \mathrm{C}$. The label was then chased with 25 -fold excess of unlabeled diferric transferrin for the indicated times (Fig. 1). Cells were chilled, collected, lysed, and the supernatants were reacted with antibody to mobilferrin and hemoglobin. Radiolabeled iron from transferrin was taken into cells. Iron was initially released from transferrin, the label was chased into hemoglobin. This established a pathway of iron transit from transferrin to mobilferrin to hemoglobin. When a similar experiment was performed with $\mathrm{NH}_{4} \mathrm{C} 1$ (Fig. $1 B$ ) the transfer of iron was inhibited. The $\mathrm{NH}_{4} \mathrm{C} 1$ inhibits the acidification of the endosome (22). Since $\mathrm{NH}_{4} \mathrm{C} 1$ has a specific effect on the endosome and inhibited the labeling of both mobilferrin and hemoglobin, the endosomal transferrin must have initiated the pathway. On the other hand, when iron was transported from iron citrate by the MIP pathway, the endosome was not involved and the addition of $\mathrm{NH}_{4} \mathrm{C} 1$ had no effect (Fig. 1, $C$ and $D$ ).

Mobilferrin is located with the clathrin-coated vesicle. The pulse chase experiments described above implicated mobilferrin in the internalization of iron from vesicles containing radioiron transferrin. Mobilferrin was initially isolated from the cytoplasmic fraction of intestinal cells (8) and was found homologous to calreticulin a protein that is usually membrane associated $(26,27)$. Western blot analysis of sucrose gradients separating membrane fractions of K562 cells demonstrated the presence of mobilferrin/calreticulin both in the cytoplasm and associated with clathrin-coated vesicles (Fig. 2). Clathrincoated vesicles are known to be associated with a large variety of proteins in addition to transferrin (28). Thus some mobilferrin was present in fractions containing the clathrin immunoreactive fractions which are associated with the transferrin containing vesicle.

Mobilferrin accepts iron from transferrin in the vesicle. K562 cells were incubated with ${ }^{59} \mathrm{Fe}$ labeled diferric transferrin and ${ }^{125}$ I-labeled diferric transferrin in the presence of $15 \mathrm{mM}$ $\mathrm{NH}_{4} \mathrm{C} 1$. The $\mathrm{NH}_{4} \mathrm{C} 1$ was used to block release of iron from transferrin in vesicles (22). After lysis of the cells, the vesicle portion was separated from the cytoplasm by $60-20 \%$ sucrose gradient (Fig. $3 \mathrm{~A}$ ). The clathrin containing vesicles were identified by Western blot demonstrating the presence of clathrin

transferrin and ${ }^{125} \mathrm{I}$ diferric transferrin at $4^{\circ} \mathrm{C}$, and then warmed to $37^{\circ} \mathrm{C}$ for $10 \mathrm{~min}$ allowing internalization of the bound transferrin. Cells were lysed and the organelles separated on a 60-20\% sucrose gradient and fractions collected. Both ${ }^{59} \mathrm{Fe}(\bullet)$ and ${ }^{125} \mathrm{I}(\boldsymbol{\Delta})$ were measured. The clathrin was located by Western blots. Fraction 1 represents the most dense portion of the sucrose gradient and the gradient ended at fraction 25. The remaining fractions were the buffer overlay representing the cytoplasmic portion of the cell lysate. $(B)$ Gel filtration of proteins removed from the vesicles. Peak tubes from the clathrin containing vesicles ( $A$, fractions 9-11) were pooled and adjusted to $1 \mathrm{M} \mathrm{NaC} 1$ to disassociate mobilferrin. Chromatography was performed with an Ultrogel AcA44 column and fractions were collected and the ${ }^{59} \mathrm{Fe}$ was quantified. The elution volume for authentic transferrin ( $\mathrm{Tf}$ ) and mobilferrin (Mb) were also obtained. The void volume of the column was at fraction 25 . 

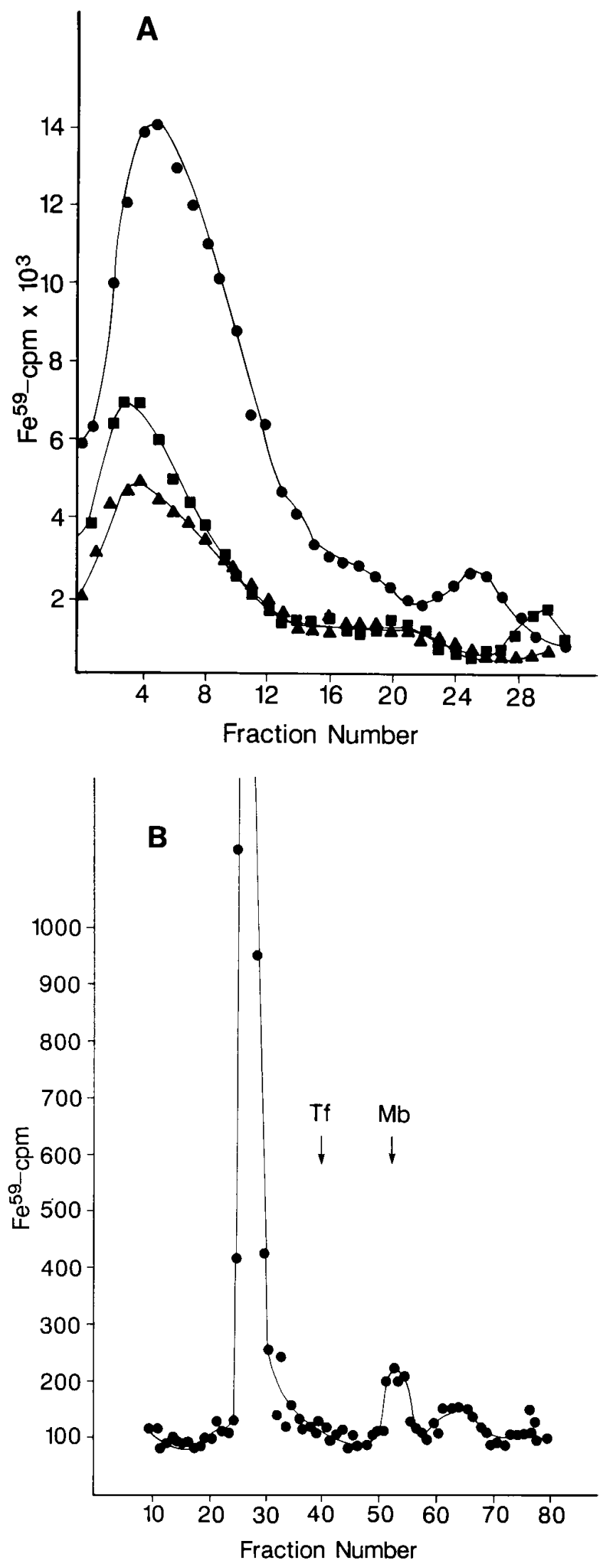

Figure 4. Iron is released from transferrin to bind mobilferrin and form end products. $(A)$ A sucrose gradient was used to separate organelles from $\mathrm{K} 562$ cells in the absence of $\mathrm{NH}_{4} \mathrm{C} 1$. Incubation, lysis and centrifugation were performed as described in Fig. 3. The ordi- (fractions 9-14). This peak contained both ${ }^{59} \mathrm{Fe}$ and ${ }^{125} \mathrm{I}$ demonstrating the coincident presence of transferrin protein and iron in these vesicles. A second peak (fractions 20-23) contained ${ }^{125} \mathrm{I}$ (and therefore the protein portion of transferrin) but proportionately much less ${ }^{59} \mathrm{Fe}$, suggesting that the transferrin had released the majority of the iron originally bound. The cytoplasm (fractions $>24$ ) had insignificant amounts of radioiron or transferrin.

The vesicles containing iron and transferrin (fractions 9-11) were incubated with $1 \mathrm{M} \mathrm{NaC1}$ to dissociate mobilferrin from the vesicles and then were placed on an Ultrogel AcA44 column. The elution of radioactive iron was determined (Fig. 3 B). Iron was found at the void volume (labeled vesicles). Small quantities of radioiron were also found at an elution volume corresponding to transferrin. In addition, radiolabeled iron was detected at a hydrodynamic volume corresponding to mobilferrin. Incubation of the vesicles with either low salt concentrations or with $4 \mathrm{M}$ urea failed to release a fraction corresponding to mobilferrin, suggesting that there was an electrostatic association between the vesicle and mobilferrin. Immunoprecipitation of the mobilferrin peak with anti-mobilferrin (data not shown) indicated that radioactive iron was present in immunoreactive mobilferrin.

Utilization of transferrin iron. K562 cells were incubated under identical conditions but in the absence of $\mathrm{NH}_{4} \mathrm{C} 1$ and cell homogenates were separated on sucrose gradients. With progressive times of incubation, iron continued to increase in the high density fraction (Fig. $4 A$ ). Accumulation of iron in this fraction (fractions 2-8) was markedly inhibited (threefold) by the specific potent inhibitor of heme synthesis succinylacetone without affecting cell viability (Trypan blue) (29). This dense fraction contains hemoglobin and/or other heme-containing proteins in various synthetic stages. In the cytoplasmic portion of the gradient (fractions 23-28) material containing ${ }^{59} \mathrm{Fe}$ was detected after $10 \mathrm{~min}$ incubation. When this material was subjected to chromatography on an Ultrogel AcA44 column (Fig. $4 \mathrm{~B}$ ) a portion of the radioactivity was found with an elution volume coincident with mobilferrin. This implied that cytoplasmic mobilferrin was labeled from transferrin iron originally derived from the vesicles; this was consistent with the pulse-chase experiments described earlier. The sucrose gradients did not demonstrate whether mobilferrin in the cytoplasm was labeled before heme synthesis or subsequently. However, the role of cytoplasmic mobilferrin as a precursor to hemoglobin was apparent in the pulse-chase studies.

\section{Discussion}

K562 erythroleukemia cells are capable of acquiring iron from tissue culture media utilizing either the transferrin-transferrin

nate shows cpm of ${ }^{59} \mathrm{Fe}$ present in the individual fractions after incubation of the $\mathrm{K} 562$ cells at $37^{\circ} \mathrm{C}$ for $0 \mathrm{~min}(\boldsymbol{\Delta}), 1$ minute $(\boldsymbol{\square})$ and 10 $\min (\bullet)$. (B) The cytoplasmic ( $A$, fractions, 23-28 after $10 \mathrm{~min}$ incubation) portion of the gradient was pooled, adjusted to $1 \mathrm{M} \mathrm{NaC1}$ and chromatographed on an Ultrogel AcA44 column as described in Fig. 3. The cpm of ${ }^{59} \mathrm{Fe}$ was determined for each fraction. Authentic samples of transferrin (Tf) and mobilferrin (Mb) eluted at the indicated fractions, and the void volume of the column was at fraction 27 . The peak observed at fraction 53 was coincident with the molecular mass of mobilferrin as exhibited on this column. 


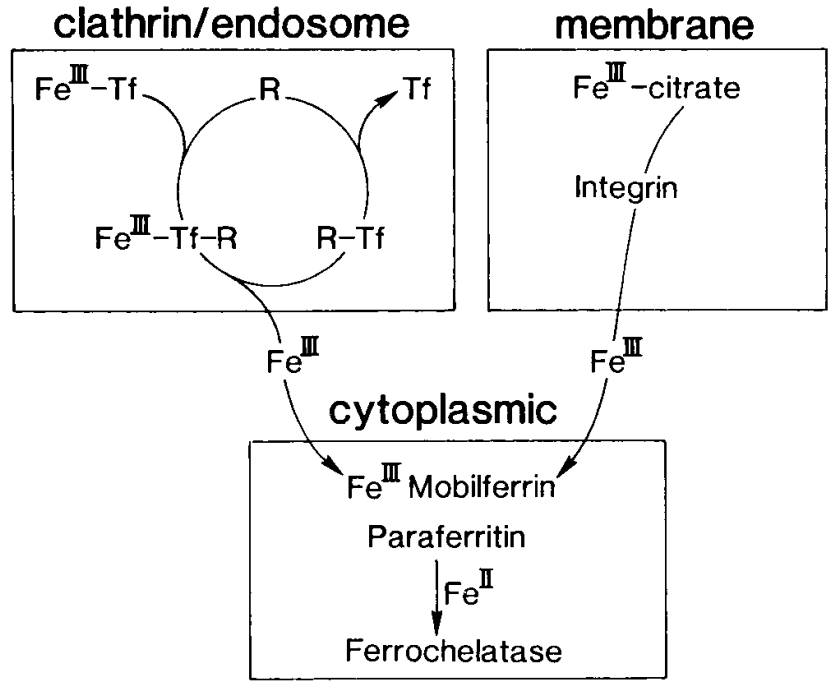

Figure 5. Schematic diagram of iron transport in K562 cells. The classic transferrin ( $\mathrm{Tf}$ ) mediated iron uptake occurs by means of a specific transferrin receptor $(\mathrm{R})$. The complex of protein and receptor are internalized in clathrin coated pits and fused with the endosome, which during acidification releases the iron. The non-transferrin pathway utilizes inorganic iron in the form of chelates, the most common of which is citrate. The iron transits the membrane in association with a cell surface integrin. The two pathways converge as mobilferrin accepts the iron. The iron is reduced, possibly by the mobilferrin containing complex paraferritin in order to be utilized by the mitochondrial protein ferrochelatase which combines the iron and porphyrin for heme synthesis. There are undoubtably other proteins and factors involved in this pathway which remain to be described.

receptor pathway or the mobilferrin-integrin pathway $(1,21)$. Iron acquired via both pathways becomes available to the cell for the production of end products such as hemoglobin and ferritin (21). Previous studies suggested that some iron presented to these cells bound to transferrin subsequently was found labeled to mobilferrin isolated from the cell cytosol. This suggested an interrelationship between the transferrintransferrin receptor pathway and the mobilferrin-integrin pathway in which mobilferrin served as a common constituent of both pathways (21). Possibly this occurs because of the close association of mobilferrin and flavin monooxygenase (30). The latter complex can serve as a NADPH dependent ferrireductase and iron must be converted to the ferrous state to be utilized for production of heme proteins.

In the current studies radiolabeled iron from transferrin was transferred to mobilferrin in K562 cells. Pulse-chase experiments showed that transferrin iron subsequently bound mobilferrin and then, in turn, hemoglobin. This processing of iron by the cell was blocked by $\mathrm{NH}_{4} \mathrm{Cl}$ which prevented acidification of the endosome (24) and delays the release of iron from transferrin internalized in the endosome. While $\mathrm{NH}_{4} \mathrm{Cl}$ dramatically suppressed the utilization of transferrin iron for labeling of mobilferrin, $\mathrm{NH}_{4} \mathrm{Cl}$ had no significant effect upon the labeling of mobilferrin when cells were incubated in radiolabeled ferric citrate. This provided assurance that radioiron was not released from transferrin before passage into the cell within the endosome. Although precise quantification can not be obtained by methods used in this study, the pulse chase experimental results suggested that a substantive portion of the iron from transferrin passed through mobilferrin before being incorporated into hemoglobin (Fig. 1). Since mobilferrin was shown by Western blots to colocalize with the endosome, it was likely that mobilferrin docked on the endosome to bind iron released from intravesicular transferrin. Subsequently the iron bound to mobilferrin became available for use by the cell with mobilferrin serving as a shuttle protein for delivery of iron in the appropriate redox state.

Due to the rapid transit, release, and metabolism of transferrin iron, these data do not exclude other, as yet, uncharacterized intermediates or an additional pathway (31). The exact mechanism by which free iron crosses the membrane from the endosome to the cytoplasm is not clear and may involve either $\mathrm{H}^{+}$ATPase (32) or a mechanism similar to the pathway described in intestinal mucosa (7). In broadest outline, a pathway for the transport and utilization of iron is beginning to emerge (Fig. 5). A multiplicity of other factors needed to control the movement and redox of iron remain to be defined.

\section{Acknowledgments}

This study was supported by the Merit Award Grant DK36612 of the National Institutes of Diabetes and Digestive and Kidney Disease of the National Insitutes of Health.

\section{References}

1. Klausner, R.D., J. van Renswoude, G. Ashwell, C. Kempf, A.N. Schechter, A. Dean, and K.R. Bridges. 1983. Receptor mediated endocytosis of transferrin in K562 cells. J. Biol. Chem. 258:4715-4724.

2. Thorstensen, K., and I. Romslo. 1990. A role of transferrin in the mechanism of cellular iron uptake. Biochem. J. 271:1-10.

3. Dickson, R.B., J.A. Hanover, M.C. Willingham, and I. Pastar. 1983. Prelysosomal divergence of transferrin and epidermal growth factor during receptor mediated endocytosis. Biochem. 22:5667-5674.

4. Crichton, R.R., and R.J. Ward. 1992. Iron metabolism-New perspectives in view. Biochem. 31:11255-11264.

5. Parmley, R.T., J.C. Barton, and M.E. Conrad. 1985. Ultrastructural localization of transferrin, transferrin receptor and iron binding sites on human placental and duodenal microvilli. Br. J. Haematol. 60:81-87.

6. Conrad, M.E., J.N. Umbreit, R.D.A. Peterson, E.G. Moore, and K.P. Harper. 1993. Function of integrin in duodenal mucosal uptake of iron. Blood. 81:517-521.

7. Conrad, M.E., and J.N. Umbreit. 1993. Iron absorption-The mucin mobilferrin integrin pathway. A competitive pathway for iron absorption. Am. J. Hematol. 42:67-73.

8. Conrad, M.E., J.N. Umbreit, E.G. Moore, R.D.A. Peterson, and M.B. Jones. 1990. A newly identified iron binding protein in duodenal mucosa of rats. Purification and characterization of mobilferrin. J. Biol. Chem. 265:52735279.

9. Muir, W.A., U. Hopfer, and M. King. 1984 Iron transport across brushborder membranes from normal and iron-deficient mouse upper small intestine. J. Biol. Chem. 259:4846-4903.

10. Brissot, P., T.L. Wright, W.L. Ma, and R.A. Weisinger. 1985. Efficient clearance of non-transferrin-bound iron by rat liver in implications for hepatic iron loading in iron overload states. J. Clin. Invest. 76:1463-1470.

11. Wright, T.L., P. Brissot, W.L. Ma, and R.A. Weisinger. 1986. Characterization of non-transferrin-bound iron clearance by rat liver. J. Biol. Chem. 261: 10909-10914.

12. Grave, E.M., J. Alexander, M. Eldridge, J.P. Kusher, D. Bernstein, and J. Kaplan. 1987. Tissue distribution and clearance kinetics of non-transferrinbound iron in the hypotransferremic mouse: a rodent model for hemochromatosis. Proc. Natl. Acad. Sci. USA. 84:3457-3462.

13. Egyed, A. 1988. Carrier mediated iron transport through erythroid cell membranes. Br. J. Haematol. 68:483-486.

14. Morgan, E. 1988. Membrane transport of non-transferrin-bound iron by reticulocytes. Biochim. Biophys. Acta. 943:428-432.

15. Wright, T.L., J.G. Fitz, R.A. Weisinger, and P. Jun. 1988. Non-transferrin-bound iron uptake by rat liver: role of membrane potential difference. $J$. Biol. Chem. 263:1842-1847.

16. Sturrock, A., J. Alexander, J. Lamb, C.M. Graven, and J. Kaplan. 1990. Characterization of a transferrin-independent uptake system for iron in HeLa cells. J. Biol. Chem. 265:3139-3145.

17. Kaplan, J., I. Jordan, and A. Sturrock. 1991. Regulation of the transfer- 
rin-independent iron transport system in cultured cells. J. Biol. Chem. 266: 2997-3004.

18. Seligman, P.A., J. Kovar, R.B. Schleicher, and E.W. Gelfand. 1991. Transferrin-independent iron uptake supports B lymphocyte growth. Blood. 78: $1526-1531$.

19. Nunez, M.T., A. Escobar, A. Ahumada, and M.G. Gonzalez-Sepulreda. 1992. Sealed reticulocyte ghosts: an experimental model for the study of $\mathrm{Fe}^{2 \mathrm{t}}$ transport. J. Biol. Chem. 267:11490-11494.

20. Inman, R.S., and M. Wessling-Resnick. 1993. Characterization of transferrin-independent iron transport in K562 cells. J. Biol. Chem. 268:8521-8528.

21. Conrad, M.E., J.N. Umbreit, E.G. Moore, C. Uzel, and M.R. Berry. 1994. Alternative iron transport pathway: Mobilferrin and integrin in K562 cells. J. Biol. Chem. 269:7169-7173.

22. Maxfield, F.R. 1985. Acidification of endocytic vesicles and lysozymes. In Endocytosis. I. Pastan, and M.C. Willingham, editors. Plenum Press. N.Y.pp 235-257.

23. Hopkins, C.R., and I.S. Trowbridge. 1983. Internalization and processing of transferrin and the transferrin receptor in human carcinoma A431 cells. $J$. Cell. Biol. 97:508-521.

24. Daiss, J.L., and T.F. Roth. 1983. Isolation of coated vesicles: Comparative studies. Methods Enzymol. 98:337-349.

25. Laemmli, U.K. 1970. Cleavage of structural proteins during assembly of the head of bacteriophage T4. Nature (Lond.). 227:680-685.

26. Conrad, M.E., J.N. Umbreit, E.G. Moore. 1993. Mobilferrin is a homologue of calreticulin. Gastroenterology. 104:1700-1704.

27. Michalak, M.I., R.E. Milmer, K. Burns, and M. Opas. 1992. Calreticulin. Biochem. J. 285:681-692.

28. Lisanti, M.P., L.S. Shapiro, N. Moskowitz, E.L. Hua, S. Puszkin, and W. Schook. 1982. Isolation and preliminary characterization of clathrin associated protein. Eur. J. Biochem. 125:463-470.

29. Ebert, P.S., R.A. Hess, B.C. Frykholm, and D.P. Tschudy. 1979. Succinylacetone, a potent inhibitor of heme biosynthesis: Effect on cell growth, heme content, and delta aminolevulinic acid dehydratase activity of malignant murine erythroleukemia cells. Biochem. Biophys. Res. Commun 88:1382-1390.

30. Umbreit, J.N., M.E. Conrad, E.G. Moore, M.P. Desai, and J. Turrens. 1996. Paraferritin: a protein complex with ferrireductase activity is associated with iron absorption in rats. Biochemistry. 35:6460-6469.

31. Hodgsonhl, E.A. Quail, E.H. Morgan. 1994. Receptor independent uptake of transferrin-bound iron by reticulocyte. Arch. Biochem. Biophys. 308: 318-326.

32. Li, C.-Y., J.A. Watkins, S. Hanazaki, J.D. Altazan, and J. Glass. 1995. Iron binding; a new function for the reticulocyte endosomal $\mathrm{H}^{+}$-ATPase. Biochemistry. 34:5130-5136. 